See Article page 189.

\section{Commentary: Belt and suspenders! Maximizing safety in central to peripheral extracorporeal membrane oxygenation conversions}

\author{
Bree Ann C. Young, MD, and Paul C. Tang, MD, PhD
}

Venoarterial extracorporeal membrane oxygenation (VAECMO) is often used as an initial temporary mechanical support strategy when faced with postcardiotomy shock as well as primary graft dysfunction after heart transplantation. Central cannulation is commonly the initial mode of access, given that patients are already cannulated in this configuration for their primary surgery. As Ohira and colleagues ${ }^{1}$ point out, thrombus formation may occur at the central aortic cannulation site. This may result from permissively subtherapeutic anticoagulation to promote hemostasis, a low flow state, and/or poor pulsatility.

The authors cite a high incidence of stroke with central cannulation as the impetus for developing a safer arterial decannulation technique to minimize the risk of embolism. ${ }^{2}$ However, it should be noted that others, including Ranney and colleagues, ${ }^{3}$ have reported a similar incidence of stroke in central versus peripheral cannulation in a retrospective cohort of 131 patients. The risk of thromboembolic stroke originating in proximity to the central aortic cannula likely varies depending on the duration of central cannulation, the anticoagulation status, the atherosclerotic burden of the aorta as a substrate for thrombosis, and the vigor of cardiac ejection that washes over the aortic cannula and cannulation site. In view of this, the creation of a theoretically safer technique for aortic decannulation with the goal of stroke prevention is much welcomed.

From the Department of Cardiac Surgery, University of Michigan, Ann Arbor, Mich. Disclosures: The authors reported no conflicts of interest.

The Journal policy requires editors and reviewers to disclose conflicts of interest and to decline handling or reviewing manuscripts for which they may have a conflict of interest. The editors and reviewers of this article have no conflicts of interest.

Received for publication Oct 2, 2020; revisions received Oct 2, 2020; accepted for publication Oct 9, 2020; available ahead of print Oct 15, 2020.

Address for reprints: Paul C. Tang, MD, PhD, Department of Cardiac Surgery, University of Michigan, Ann Arbor, MI (E-mail: tangpaul@med.umich.edu).

JTCVS Techniques 2020;4:193-4

2666-2507

Copyright (C) 2020 The Authors. Published by Elsevier Inc. on behalf of The American Association for Thoracic Surgery. This is an open access article under the CC BY-NCND license (http://creativecommons.org/licenses/by-nc-nd/4.0/).

https://doi.org/10.1016/j.xjtc.2020.10.011

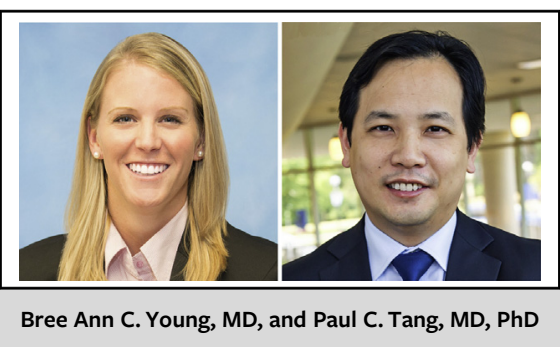

CENTRAL MESSAGE

Access denial for emboli to the cerebral vasculature while maintaining cerebral perfusion may be an effective method for lowering stroke risk associated with aortic decannulation from central extracorporeal membrane oxygenation.

Ohira and colleagues describe brief occlusion of the left subclavian and head vessels to deny access to the cerebral circulation from any potential thrombus that dislodges during aortic decannulation. The right carotid is kept perfused during this procedural sequence from perfusion through the right axillary artery arterial access. Flow exiting from the innominate artery then washes the aortic cannulation site. An simpler alternative protocol in which only the left carotid is isolated and briefly occluded may yield comparable results. Blood exiting the innominate artery should be sufficient to preclude embolism into the right cerebral circulation. Because the cerebral circulation is interrupted only briefly (90 seconds or less), the risk of permanent neurologic injury is likely low, particularly when paired with unilateral antegrade cerebral perfusion. However, it should be noted that this is normothermic arrest of the cerebral circulation, and blood flow should be allowed to return expeditiously. The question remains whether a brief unilateral or bilateral interruption of cerebral flow without cerebral perfusion is safe when converting from central to femoral cannulation.

Unfortunately, a large dataset showing benefit or otherwise is not available, given the novel nature of this technique. The need to expose head vessels may dampen enthusiasm for those less familiar with aortic arch procedures; however, simplifying the approach to only clamping the left carotid should be less taxing and may encourage wider adoption. Only then can we accumulate sufficient experience to examine this in a robust manner. Although 
only limited data exist, novel surgical techniques that may optimize ECMO outcomes with low risk for harm should be embraced and added to the repertoire of surgeons working with this critically ill population.

\section{References}

1. Ohira S, Spielvogel D, Lansman SL, Kai M. Unilateral antegrade cerebral perfusion using axillary venoarterial extracorporeal membrane oxygenation during central decannulation. J Thorac Cardiovasc Surg Tech. 2020;4: 189-92.

2. Mariscalco G, Salsano A, Fiore A, Dalén M, Ruggieri VG, Saeed D, et al. Peripheral versus central extracorporeal membrane oxygenation for postcardiotomy shock: multicenter registry, systematic review, and meta-analysis. J Thorac Cardiovasc Surg. 2020;160:1207-16.

3. Ranney DN, Benrashid E, Meza JM, Keenan JE, Bonadonna DK, Bartzet R, et al. Central cannulation as a viable alternative to peripheral cannulation in extracorporeal membrane oxygenation. Semin Thorac Cardiovasc Surg. 2017; 29:188-95. 